\title{
Prevalence of antibodies to poliovirus in 1978 among subjects aged $0-88$ years
}

\author{
MARY ROEBUCK, ROMA CHAMBERLAIN
}

\begin{abstract}
The antibody state of a population aged 6 months to 88 years to poliovirus types 1,2 , and 3 was determined by examining 919 sera collected in Lancashire, London, and southern and south-east England. In subjects aged over 2 years the immune state was surprisingly uniform, although the older patients had probably acquired practically all their antibodies as a result of natural infection and those under 16 through vaccination. At least $95 \%$ had detectable antibodies to at least one poliovirus type and about $60 \%$ to all three types, with the exception of a cohort of children born between 1963 and 1968, in whom the proportions were about $80 \%$ and $40 \%$ respectively. These children were born around the time of the changeover from inactivated to oral vaccine, when immunisation rates were low and there was confusion over the number of doses required.

These results indicate that a complete course of vaccine or a booster dose at or around school-leaving age is necessary.
\end{abstract}

\section{Introduction}

After the highly successful vaccination campaign which resulted in the disappearance of epidemic paralytic poliomyelitis general interest in the susceptibility of the population to poliomyelitis in the United Kingdom waned. Mortimer and Cunningham, ${ }^{1}$ however, after examining sera collected during 1972-4 from children aged 0-14 years, suggested that the proportion of the young population with antibody to all three types of poliovirus and the current acceptance rates for oral poliovirus were barely satisfactory. At about the same time there was concern that vaccination rates might fall permanently because of the public

Central Public Health Laboratory, London NW9 5HT

MARY ROEBUCK, MB, DIPBACT, senior virologist, Virus Reference Laboratory

ROMA CHAMBERLAIN, MB, FFCM, senior epidemiologist, Epidemiology Research Laboratory reaction against pertussis immunisation. Codd and White, ${ }^{2}$ examining the sera of 1016 patients aged $0-60$ collected during 1976-7, suggested that the immune state of the population in the north of England, particularly those under 20, was unsatisfactory and that "the introduction of wild polio strains, given suitable seasonal and environmental conditions, is likely to lead to outbreaks and an increase in the incidence of paralytic disease."

During 1978, 131 strains were examined at the Virus Reference Laboratory, Colindale, four (3\%) of which possessed marker characteristics of wild virus. It is not unreasonable to assume that neurovirulent strains of poliovirus are introduced occasionally by travellers from countries where poliomyelitis is endemic and circulate among the population. It is, therefore, important to ensure that adequate levels of immunity are maintained throughout the community.

Although close surveillance is kept on all cases of poliomyelitis and on use of the vaccine, little has been published about the overall state of seroimmunity in various age groups. The aim of our study was to investigate the level of immunity at different ages.

\section{Materials and methods}

Sera-Altogether 928 sera were examined, of which 782 had originally been sent for estimation of antistreptolysin 0 titre. These samples came from southern and south-east England. The remaining 146 samples had been referred to the Virus Reference Laboratory from Lancashire and the London area for estimations of antibody to enteroviruses. None of the sera in this group came from patients with neurological disease or suspected polio. All specimens were collected during 1978 and were diluted one in eight and stored at $-30^{\circ} \mathrm{C}$ until required.

Viruses-Sabin poliovirus types 1,2 , and 3 were used, a preliminary test having indicated that the responses to wild virus and Sabin virus did not appear to differ. The viruses were grown in Hep2C tissue culture in $125 \mathrm{ml}(4 \mathrm{oz})$ bottles, harvested when maximum cytopathogenic effect was attained, and titrated in microtitre plates to establish the tissue culture infecting dose. In the test 100 times the tissue culture infecting dose of each serotype was used. The titrated viruses were stored in aliquots of $0.5 \mathrm{ml}$ at $-30^{\circ} \mathrm{C}$.

Tissue culture-Hep2C cells were grown in $125 \mathrm{ml}$ bottles in a growth medium consisting of Eagles Minimum Essential Medium plus 5\% newborn calf serum, $2 \%$ of $4.4 \%$ sodium bicarbonate, and $0.1 \%$ of Crystamycin (benzylpenicillin sodium and streptomycin sulphate; 
$10^{8}$ units/l). On the day of the test the monolayer was trypsinised and the contents of one $125 \mathrm{ml}$ bottle suspended in $20 \mathrm{ml}$ of growth medium.

Serum neutralisation tests-The sera were screened at a dilution of 1 in $\mathbf{8}$ for their ability to neutralise 100 times the tissue culture infecting dose of each poliovirus serotype. The antibody estimations were performed in microtitre plates. One plate was used for each of the poliovirus types, and one drop of serum dilution ( 1 in 8 ) was distributed into three wells per microtitre plate. One drop of virus suspension (100 times the tissue culture infecting dose) was then added to all the wells. The plates were covered and incubated at $37^{\circ} \mathrm{C}$ in $5 \%$ carbon dioxide for four to five hours, after which a volume of Hep2C cell suspension was added and the plates re-covered and returned to the incubator for a further five days, after which they were read. Standard antiserum controls were set up with each batch.

\section{Results}

\section{SEROIMMUNITY}

Nine children aged under 6 months were excluded from this analysis because of the possible presence of residual maternal antibodies, leaving 919 sera from subjects aged between 6 months and 88 years. Figure 1 shows the proportion of sera in each age group with antibody titres of $1 / 8$ to at least one poliovirus type ("partial protection") and the proportion with antibody to all three types ("complete protection").

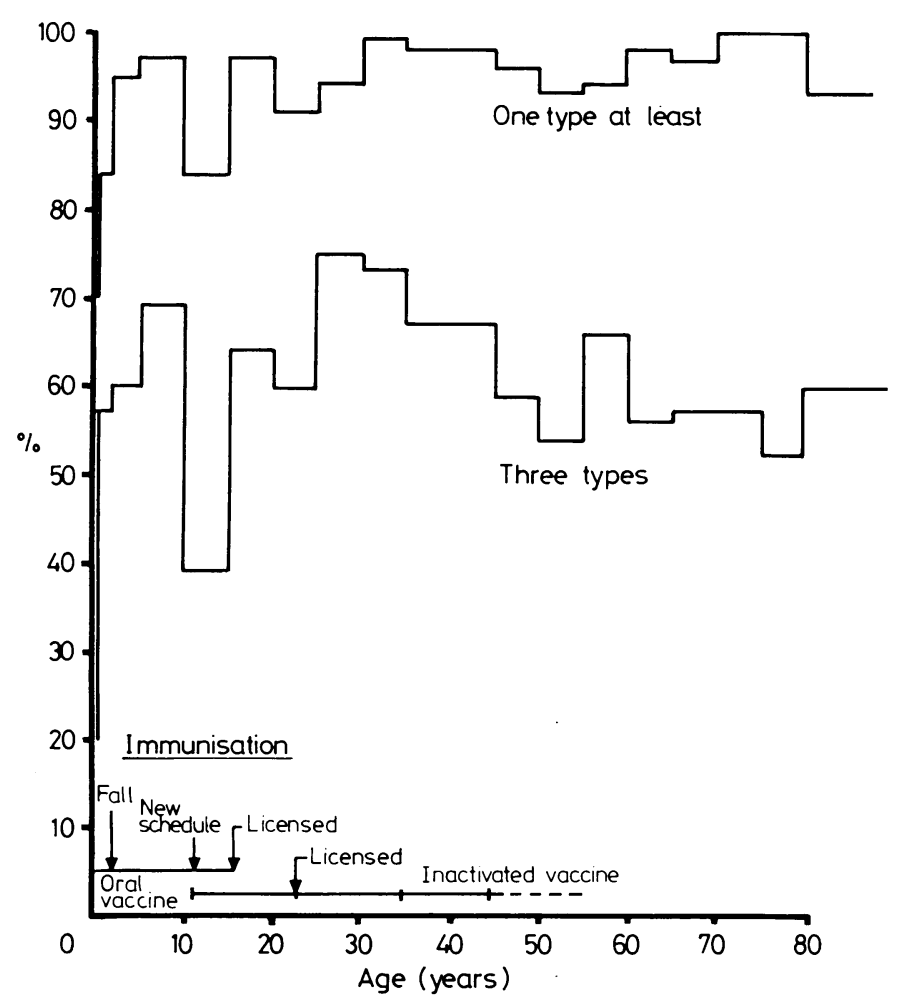

FIG 1-Proportions of subjects with antibodies to all three polioviruses or at least one type according to age.

In subjects aged up to 25 years the ratio of complete to partial protection remained about the same. In subjects aged between 25 and 50 the proportion with partial protection remained high but the proportion with complete protection declined gradually with increasing age. In subjects aged 10-15 years proportions with both complete and partial protection were lower. This appeared so exceptional that we tested a further 200 samples collected in 1976 and 1980 from children born between 1961 and 1970. Although the proportion with antibodies was slightly higher, the results confirmed that a cohort of children born at this time were not as well protected as those younger or older.

Figure 2 shows the proportion of sera examined with antibodies to each of the poliovirus types. While the patterns for poliovirus types 1 and 2 closely resembled each other, that for poliovirus type 3 was

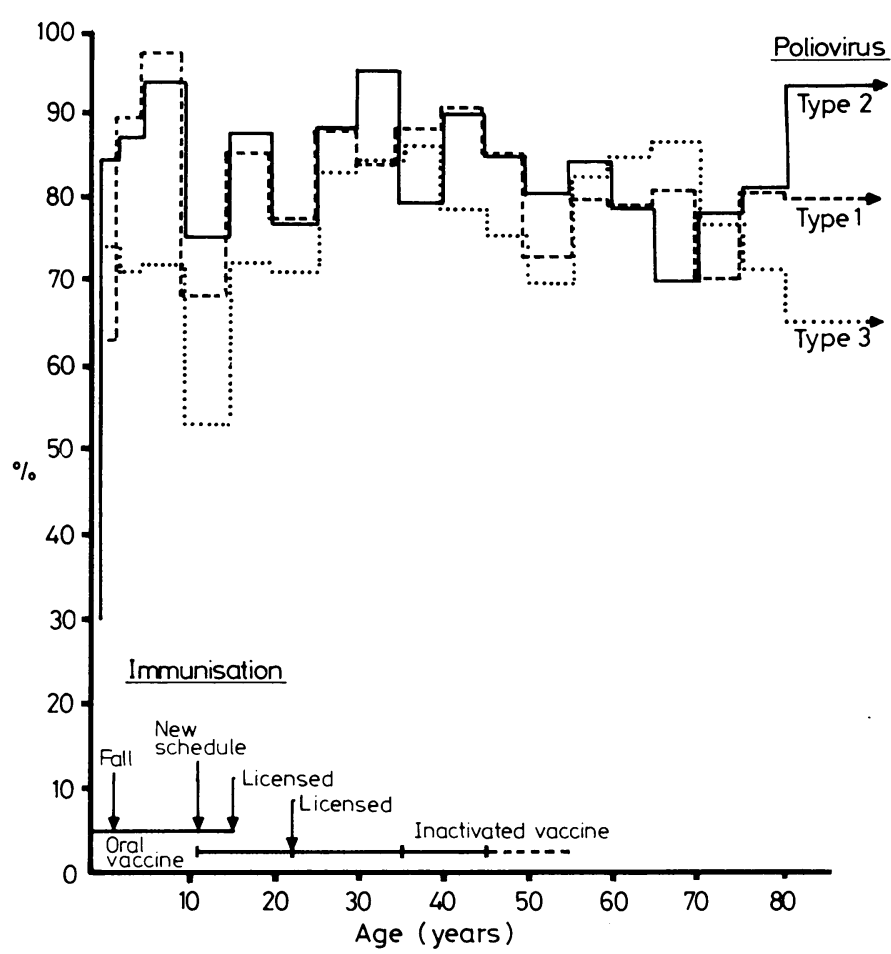

FIG 2-Proportions of subjects with antibodies to each poliovirus type according to age.

different. In subjects aged under about 50 years and especially in those under 30 the proportion with antibody to type 3 was lower than for the two other types, and in general this determines the level of complete protection.

RELATION OF PREVALENCE OF ANTIBODIES TO NATURALLY OCCURRING POLIOMYELITIS AND VACCINATION

The proportion of serum antibody detected in the population reflects the occurrence of natural infection and the extent of immunisation.

In May 1956 Salk inactivated vaccine became available in Britain, and in 1957 it was offered to those born from 1943 onwards, later extended to 1933 . By 1963 it was estimated that complete courses had been given to $83 \%$ of people born between 1943 and $1962,65 \%$ of those born between 1933 and 1942, and about $46 \%$ of those of other ages. There was, however, concern about the potency of the vaccine in relation to poliovirus type 1 . In February 1962 Sabin oral vaccine was introduced. Courses already started using Salk vaccine were sometimes completed with oral vaccine, and there was confusion about the number of doses needed. In May 1963 it was recommended that those who had already received two doses of Salk vaccine should be given two doses (not one) of Sabin vaccine followed by a reinforcing dose at 5 years of age. At that time polio vaccine was given separately from other immunisations.

As the number of cases of poliomyelitis fell interest in vaccination waned, and in 1966 the chief medical officer of the Ministry of Health expressed concern about the poor uptake of polio vaccine. In 1967 a simpler combined schedule of diphtheria, tetanus, and pertussis and polio immunisation was introduced starting at the age of about 6 months. The uptake improved, and from then onwards about $80 \%$ of children under the age of 2 years received a full course, although in some areas the proportion was far short of this. For a short period after 1974 the rates dropped slightly, but by 1978 they had recovered.

Table I presents our findings according to the method of vaccination in use at the time of birth of the subjects. It may be seen that, with the exception of two groups, partial protection was high and unaffected by age. Complete protection, however, was about $10 \%$ higher among those who might have been immunised (by either oral or inactivated poliovirus vaccine) than those who had probably acquired their immunisation through natural exposure. The exceptions were children under 2 years, many of whom would not have completed their courses, and those aged 10-15 years, who were born between 1963 and 1968- 
TABLE I-Proportions of subjects with antibodies to all three polioviruses or to at least one type according to age and use of vaccine

\begin{tabular}{lccccc}
\hline Year of birth & Age & $\begin{array}{c}\text { Total } \\
\text { No of } \\
\text { subjects }\end{array}$ & $\begin{array}{c}\text { No }(\%) \\
\text { completely } \\
\text { protected }\end{array}$ & $\begin{array}{c}\text { No }(\%) \\
\text { partially } \\
\text { protected }\end{array}$ & $\begin{array}{c}\text { Immunisation } \\
\text { history }\end{array}$ \\
\hline $1978-1976$ & 6 months- & 19 & $11(58)$ & $16(84)$ & Incomplete OPV* \\
$1976-1968$ & 2 years- & 150 & $98(65)$ & $144(96)$ & OPV \\
$1968-1963$ & 10 years- & 75 & $29(39)$ & $63(84)$ & IPV+ OPV \\
$1963-1943$ & 15 years- & 227 & $155(68)$ & $219(96)$ & $83 \%$ IPV \\
$1943-1933$ & 35 years- & 110 & $74(67)$ & $108(98)$ & $65 \%$ IPV \\
$1933-1913$ & 45 years- & 228 & $133(58)$ & $218(96)$ & \\
$1913-1890$ & $65+$ & 110 & $62(56)$ & $108(98)$ & \\
\hline
\end{tabular}

$\mathrm{OPV}=$ Oral poliovirus vaccine. $\mathrm{IPV}=$ Inactivated poliovirus vaccine

*Includes children who had both completed and partially completed courses.

Includes children who had received primary and booster doses at school entry. †Includes some children who had received primary courses and booster doses a school entry and at school-leaving age.

that is, at the time of the changeover from inactivated to oral vaccine, the confusion over the number of doses required, and the introduction of the combined diphtheria, tetanus, and pertussis and polio schedules.

\section{POLIOVIRUS STRAINS}

Table II shows the proportions of subjects who had antibodies to each of the three poliovirus types regrouped as in table I. Overall, the proportions with antibodies to poliovirus type 3 were lower than for the two other types. The difference was not great among the older patients, but among children born after the introduction of oral vaccine the proportion with antibodies to poliovirus types 1 and 2 was $91-93 \%$ whereas for poliovirus type 3 it was only $72 \%$. None of the other age groups were as well protected against types 1 and 2 .

TABLE II-Proportions of subjects with antibodies to poliovirus types 1, 2, and 3 according to age and use of vaccine

\begin{tabular}{|c|c|c|c|c|c|c|c|c|c|}
\hline \multirow{3}{*}{ Year of birth } & \multirow{3}{*}{ Age } & \multirow{3}{*}{$\begin{array}{c}\text { Total } \\
\text { No of } \\
\text { subjects }\end{array}$} & \multicolumn{6}{|c|}{ No $(\%)$ with antibodies to: } & \multirow{3}{*}{$\begin{array}{c}\text { Immunisation } \\
\text { history }\end{array}$} \\
\hline & & & \multicolumn{2}{|c|}{ Type 1} & \multicolumn{2}{|c|}{ Type 2} & \multicolumn{2}{|c|}{ Type 3} & \\
\hline & & & No & $\%$ & No & $\%$ & No & $\%$ & \\
\hline $\begin{array}{l}1978-1976 \\
1976-1968 \\
1968-1963 \\
1963-1943 \\
1943-1933 \\
1933-1913 \\
1913-1890\end{array}$ & $\begin{array}{l}6 \text { months- } \\
2 \text { years- } \\
10 \text { years- } \\
15 \text { years- } \\
35 \text { years- } \\
45 \text { years- } \\
65+\end{array}$ & $\begin{array}{r}19 \\
150 \\
75 \\
227 \\
110 \\
228 \\
110\end{array}$ & $\begin{array}{r}12 \\
140 \\
51 \\
191 \\
98 \\
181 \\
85\end{array}$ & $\begin{array}{l}63 \\
93 \\
68 \\
84 \\
89 \\
79 \\
77\end{array}$ & $\begin{array}{r}16 \\
136 \\
56 \\
200 \\
93 \\
187 \\
86\end{array}$ & $\begin{array}{l}84 \\
91 \\
75 \\
88 \\
85 \\
82 \\
78\end{array}$ & $\begin{array}{r}14 \\
108 \\
40 \\
177 \\
90 \\
177 \\
84\end{array}$ & $\begin{array}{l}74 \\
72 \\
53 \\
78 \\
82 \\
78 \\
76\end{array}$ & $\begin{array}{c}\text { Incomplete OPV* } \\
\text { OPV† } \\
\text { IPV and OPV } \ddagger \\
83 \% \text { IPV } \ddagger \\
65 \% \text { IPV }\end{array}$ \\
\hline
\end{tabular}

OPV $=$ Oral poliovirus vaccine. $I P V=$ Inactivated poliovirus vaccine.

*Includes children who had both completed and partially completed primary courses. Includes children who had received primary and booster doses at school entry. IIncludes some children who had receive

\section{Discussion}

This study has shown that, apart from in children under the age of 2 years and a cohort born between 1963 and 1968, the immune state to poliomyelitis is surprisingly uniform even though those over 45 years of age probably acquired their antibodies after natural infection and those under 16 from vaccination.

The sera were originally sent for many different reasons for estimations of antistreptolysin 0 titres and of antibody to enteroviruses. Those yielding negative as well as those yielding positive results were included. The subjects were of all ages and came from a wide geographical area mainly in the south of England, but as samples from hospitals may not be entirely representative the effect of any bias such as social class has to be considered.

Studies of the 1947 poliomyelitis epidemic carried out nationally $^{3}$ and in London ${ }^{4}$ showed that there was no association with raised infant mortality, which is a good social indicator, or with social class. Fallon ${ }^{5}$ showed in 1954-5 that the antibody levels in children aged 0-14 years living in a rural area in north Wales where no cases of poliomyelitis had been notified between 1915 and 1955 were almost the same as those among children of the same age living in Liverpool, where the disease was endemic.

When the older patients acquired their infection can never be known. Just over a half of the sample were born before the second world war (including a tenth who were born before the first world war), and their antibody patterns reflect the results of one or more exposures to poliovirus during their lives. Their present socioeconomic state is unlikely, therefore, materially to affect these results.

This study shows that among the younger age groups the proportion who had detectable antibodies at a dilution of $1 / 8$ to all three types of poliovirus was lower than the proportion who according to national statistics might have been expected to have received full courses of vaccine, although the proportion with antibodies to just one or two types was much higher. National statistics, however, refer to completed programmes, and those with antibodies to only one or two types may include those who did not finish a course. This suggestion is in line with the findings of the two national cohort studies-the British Births Child Study $^{6}$ and the National Child Development Study ${ }^{7}$-in which in each of the cohorts $95 \%$ of the children born in 1970 and in 1958 were stated by their parents to have had at least one dose of vaccine. In the National Child Development Study the socialclass differences were analysed: $99 \%$ of those in social class I, $97 \%$ in classes II and III NM, 96\% in class III M, 94\% in class IV, and $90 \%$ in class V were immunised. As we found that $96 \%$ of the 2- to 10-year-old group showed evidence of antibody to at least one strain of poliovirus a social-class bias, if any, is unlikely to have affected the results.

Possibly there has been a widespread infection caused by the excretion of Sabin strains after immunisation leading to partial protection of most of the unimmunised population. There was no gradual rise in antibody levels with age, however, and the presence of a small cohort of poorly protected children born between 1963 and 1968 suggests that this is unlikely to be the full explanation.

Lamy et $a l,{ }^{8}$ reporting on the prevalence of polio neutralising antibodies among all ages in Belgium for the years 1976-8, found a similar pattern, but they attributed the low figures found in children aged around 11 and 12 years to a waning of demonstrable antibody after vaccination. Their results, however, showed some increase in the proportion of children without antibodies in a wider age group between 9 and 14 years, with little difference in the proportions found in those who were either younger or older. In Belgium there was a mass campaign using Salk vaccine in 1958, live vaccine was introduced in 1963, and trivalent Sabin became obligatory in 1967. Thus the children aged 9-14 years would have been immunised at the time of the changeover from Salk to Sabin vaccine.

Mortimer and Cunningham, ${ }^{1}$ analysing serum samples taken in $1972-4$, found a lower proportion of children with no detectable antibodies among those aged 5 to 14 years compared with those aged under 5 years. They suggested that serum antibodies induced by oral vaccine did not persist as long as those induced by inactivated vaccine. Unfortunately, a finer breakdown was not published and whether the low proportion was evenly distributed throughout the 10-year age span was not stated.

In a survey carried out in Bristol in 1976 on children born in 1961, 1965, and 1969 the proportions with antibodies at a $1 / 8$ titre were all higher than our results. ${ }^{9}$ Children born in 1961 had lower primary immunisation rates than those born in 1965, but a higher proportion had antibodies to polio (virus types 1 and 3). The immunisation rates and the proportion with antibodies to each of the three polio strains were higher among those born in 1969 compared with those born in 1965. The proportion who received reinforcing doses increased progressively in each of the three years. The immunisation rates were high, but neither the type of vaccine nor the schedules used were specified.

In another survey $40 \%$ of children born in 1967 in Sydney, Australia, were found to have antibodies at a 1/8 titre to all three poliovirus types. ${ }^{10}$ This proportion was lower than the proportion 
among subjects sampled in 1976 at the age of 18 years who had received Salk vaccine. The authors attributed the decline in the proportion with antibodies to the waning of immunity to poliovirus after oral vaccine.

Thus evidence from various sources suggests that a cohort of children born in the middle 1960s has proportionately fewer circulating antibodies than those who are older or younger. In our view this is associated with a decline in the vaccination rates together with the interruption of vaccine courses brought about by changes from inactivated to oral vaccine and confusion over the number of doses needed. This does not rule out a possible waning in immunity after oral vaccine but does suggest that this may not be the only cause. The existence of this cohort of children, who are not so well protected as the rest of the population and who might contract poliomyelitis if they travel abroad or come in close contact with vaccinated babies who are excreting the virus, highlights the need for a complete course of vaccine or for a booster dose to be given at or around schoolleaving age.

The description of the incidence of poliomyelitis and the development and use of vaccine was taken from the annual reports of the Local Government Board, the Ministry of Health, and the Department of Health and Social Security.

\section{References}

${ }^{1}$ Mortimer PP, Cunningham P. Sero-immunity to poliovirus in children and young women: England 1972-4. F Hyg $1975 ; 74: 283-7$.

2 Codd AA, White E. Protection against poliomyelitis. Lancet 1977;ii: 1078.

${ }^{3}$ Hill AB, Martin WJ. Poliomyelitis and the social environment. Br Med $\mathcal{Y}$ 1949 ;ii:357-8.

${ }^{4}$ Daley A, Benjamin B. An epidemiological note on the outbreak of poliomyelitis in London in 1947. Medical Officer 1948;80:171-6.

${ }^{5}$ Fallon RJ. Serological epidemiology of poliomyelitis. Distribution of immunity to poliomyelitis virus. Lancet 1956;i:65-9.

${ }^{6}$ Chamberlain RN, Simpson RN. The prevalence of illness in childhood. Tunbridge Wells: Pitman Medical in association with the Fellowship of Postgraduate Medicine, 1979.

${ }^{7}$ Davie R, Butler N, Goldstein H. From birth to seven. A report of the National Child Development Study. London: Longman, 1972.

${ }^{8}$ Lamy ME, Cornu C, Desmyter J. Poliovirus antibodies in age groups an assessment of obligatory vaccination in Belgium. In: International Association of Biological Standardization, ed. International symposium on immunisation: benefit versus risk factors Brussels 1978. Basle: S Karger, 1979:207-13. (Developments in Biological Standardization No 43.)

${ }^{9}$ Bainton D, Freeman M, Magrath DI, Sheffield F, Smith JWG. Immunity of children to diphtheria, tetanus, and poliomyelitis. $B r$ Med 71979 ;i: 854-7.

${ }^{10}$ Menser MA, Collins E, Wu SW. Childhood immunisation 1979. Disturbing statistics for metropolitan Sydney. Med F Aust 1980;ii:131-57.

(Accepted 9 November 1981)

\title{
Hypoparathyroidism and hypocalcaemia during treatment for acute leukaemia
}

\author{
D B FREEDMAN, M SHANNON, P DANDONA, H G PRENTICE, A V HOFFBRAND
}

\begin{abstract}
Nine patients with acute leukaemia developed a complex syndrome of hypocalcaemia and hypomagnesaemia during treatment. These metabolic abnormalities developed rapidly in some patients and gave rise to acute symptoms. Immunoreactive parathormone concentrations in these patients were found to be either absolutely or inappropriately low.
\end{abstract}

Hypomagnesaemia and the action of chemotherapeutic agents, especially adriamycin or cytarabine, or both may have contributed to the pathogenesis of this syndrome.

\section{Introduction}

Hypokalaemia and hyponatraemia are recognised complications of acute leukaemia, particularly during treatment. ${ }^{1}$ We recently observed that very low plasma concentrations of calcium and

\footnotetext{
Metabolic Unit, Department of Chemical Pathology, Royal Free Hospital and School of Medicine, London NW3 2QG

D B FREEDMAN, $M B$, BS, registrar

P DANDONA, DPHIL, MRCP, director of metabolic unit and senior lecturer in chemical pathology

Academic Department of Haematology, Royal Free Hospital and School of Medicine, London NW3 2QG

$M$ SHANNON, MB, CHB, senior registrar

H G PRENTICE, MRCP, MRCPATH, senior lecture

A V HOFFBRAND, DM, FRCPATH, professor
}

magnesium are also common in patients with acute leukaemia during chemotherapy. These metabolic abnormalities present considerable management problems.

\section{Patients and methods}

During a nine-month period nine patients (eight female, one male; age range 10-81 years) undergoing intensive chemotherapy and radiotherapy including bone-marrow transplantation developed profound hypocalcaemia resulting in tetany in four. Before treatment all had been normocalcaemic and normomagnesaemic and had had normal renal function. Six of the patients had acute myeloblastic leukaemia (including two with acute myelofibrosis) and three acute lymphoblastic leukaemia (two T-cell, one common).

Eight of the nine patients had been resistant to or had relapsed from previous chemotherapy and had been referred for experimental treatment. At the time of onset of hypocalcaemia all were receiving their second or subsequent course of chemotherapy. Three of the patients had undergone allogeneic bone-marrow transplantation and thus had been exposed to total body irradiation and high-dose cyclophosphamide. Seven of the patients were receiving non-absorbable antibiotics (neomycin, colistin) for gastrointestinal decontamination and oral antifungal agents. All patients also received prophylactic co-trimoxazole.

All the patients suffered from moderate to severe vomiting and diarrhoea. Nutritional state measured by weight loss $(>10 \%$ body weight) was substantial in seven patients. None of the nine patients, however, had received parenteral nutrition.

Chemotherapeutic agents common to all nine patients were an anthracycline antibiotic (doxorubicin in eight and daunorubicin in one) and cytarabine. Six patients also received thioguanine. Other agents used in some but not all were etoposide, 2'deoxycoformycin, colaspase, vindesine, mAMSA, and methotrexate.

During the study period seven patients had proved bacterial 